\title{
Methylation Status of Imprinted Genes and Repetitive Elements in Sperm DNA from Infertile Males
}

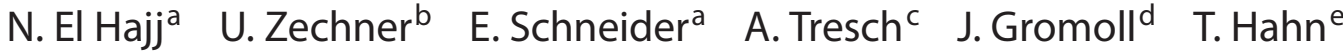 \\ M. Schorsch ${ }^{\text {e T. Haaf }}{ }^{\mathrm{a}}$ \\ a Institute of Human Genetics, Julius Maximilians University, Würzburg, bInstitute of Human Genetics, \\ Johannes Gutenberg University, Mainz, ' ${ }^{\circ}$ Gene Center and Department of Biochemistry, Ludwig Maximilians \\ University, München, ${ }^{d}$ Centre of Reproductive Medicine and Andrology, University of Münster, Münster, and \\ eFertility Center, Wiesbaden, Germany
}

\section{Key Words}

ALU repeats $\cdot$ ART outcome $\cdot$ Imprinted genes $\cdot$ Male infertility $\cdot$ Sperm DNA methylation

\begin{abstract}
Stochastic, environmentally and/or genetically induced disturbances in the genome-wide epigenetic reprogramming processes during male germ-cell development may contribute to male infertility. To test this hypothesis, we have studied the methylation levels of 2 paternally (H19 and GTL2) and 5 maternally methylated (LIT1, MEST, NESPAS, PEG3, and SNRPN) imprinted genes, as well as of ALU and LINE1 repetitive elements in 141 sperm samples, which were used for assisted reproductive technologies (ART), including 106 couples with strictly male-factor or combined male and female infertility and 28 couples with strictly female-factor infertility. Aberrant methylation imprints showed a significant association with abnormal semen parameters, but did not seem to influence ART outcome. Repeat methylation also differed significantly between sperm samples from infertile and presumably fertile males. However, in contrast to imprinted genes, ALU methylation had a significant impact on pregnancy and live-birth rate in couples with male-factor or combined infertility. ALU methylation was significantly high-
\end{abstract}

er in sperm samples leading to pregnancy and live-birth than in those that did not. Sperm samples leading to abortions showed significantly lower ALU methylation levels than those leading to the birth of a baby.

Copyright $\odot 2011$ S. Karger AG, Base

Gametogenesis is a highly complex process that involves dramatic epigenetic changes [Reik et al., 2001; Haaf, 2006]. In the mammalian germline, genome-wide demethylation erases essentially all methylation patterns in primordial germ cells around the time when the cells enter the gonadal ridge to ensure an equivalent epigenetic state in germ cells of both sexes [Hajkova et al., 2002]. Parent-specific methylation patterns according to the sex of the germline are then established during germ-cell differentiation. In the male germline, remethylation is initiated after prenatal mitotic arrest and completed postnatally during pachy tene [Marchal et al., 2004; Oakes et al., 2007]. Experimental demethylation of male germ cells in neonatal and adult mice interfered with spermatogenesis and pregnancy outcome, indicating the biological significance of germ-cell methylation [Raman and Narayan, 1995; Kelly et al., 2003]. A number of recent studies reported epigenetic abnormalities, mostly aberrant meth-

\section{KARGER}

Fax +4161306 1234

E-Mail karger@karger.ch

www.karger.com
(C) $2011 \mathrm{~S}$. Karger AG, Basel

$1661-5425 / 11 / 0052-0060 \$ 38.00 / 0$

Accessible online at:

www.karger.com/sxd
Prof. Thomas Haaf

Institute of Human Genetics, Biocenter, Julius Maximilians University Am Hubland

DE-97074 Würzburg (Germany)

Tel. +49 931318 8738, Fax +49931 318 4069, E-Mail thomas.haaf@uni-wuerzburg.de 
ylation values of imprinted genes in sperm of infertile males [Marques et al., 2004, 2008; Houshdaran et al., 2007; Kobayashi et al., 2007, 2009; Boissonnas et al., 2010; Hammoud et al., 2010; Poplinski et al., 2010].

Here we used bisulfite pyrosequencing to quantify the methylation levels of functionally important differentially methylated regions (DMRs) in imprinted genes. Genomic imprinting is a parent-specific epigenetic modification in which allele-specific methylation and expression depends on male versus female germline transmission. Many imprinted genes play essential roles in development, notably in the regulation of fetal and placental growth and tissue differentiation [Bartolomei and Tilghman, 1997; Reik et al., 2003]. Because imprinted genes escape epigenetic reprogramming after fertilization and maintain their parent-specific germline patterns, abnormal methylation imprints can be transmitted directly from the father's sperm into the developing embryo [Kobayashi et al., 2009]. In this light, they represent primary candidates when searching for the source of fertility problems and/or developmental failures occurring after ART.

In addition, we have analyzed the methylation of interspersed ALU and LINE1 repeats, which comprise $10 \%$ and $17 \%$ of the human genome [International Human Genome Sequencing Consortium, 2004], respectively, and, therefore, can indicate epigenetic changes affecting multiple loci. ALUs are non-autonomous, small elements that require the enzymatic machinery provided by LINE1 expression for retrotransposition [Hagan et al., 2003]. In somatic tissues most retrotransposon-derived elements are densely methylated to prevent retrotransposition activity [Yoder et al., 1997]. Genome-wide demethylation in primordial germ cells opens a window for transcriptional activity and even retrotransposition, but most retrotransposon-derived elements become remethylated during later stages of germ-cell development [Li, 2002]. Interestingly, a substantial fraction of ALUs is less methylated in sperm than in oocytes and somatic tissues [Rubin et al., 1994; Schmid, 1998], suggesting that the silencing process in spermatogenesis is imperfect and incomplete [Whitelaw and Martin, 2001].

\section{Materials and Methods}

\section{Sperm Samples}

Semen samples (excess materials after ART) were obtained with informed consent of the donors from 141 males undergoing infertility treatment at the Fertility Center Wiesbaden. Semen analysis was performed according to WHO guidelines. Male-fac-
Table 1. Methylation level of the studied loci

\begin{tabular}{|c|c|c|}
\hline \multirow[t]{2}{*}{ Gene/repeat } & \multicolumn{2}{|c|}{ Methylation level (mean and SD) } \\
\hline & $\begin{array}{l}\text { infertile males, } \% \\
(\mathrm{n}=106)\end{array}$ & $\begin{array}{l}\text { controls, } \% \\
(\mathrm{n}=28)\end{array}$ \\
\hline$B O L L$ & $5.5 \pm 2.9$ & $5.4 \pm 2.1$ \\
\hline HI9 & $92.3 \pm 3.5$ & $93.1 \pm 2.2$ \\
\hline GTL2 & $92.8 \pm 2.5^{*}$ & $93.8 \pm 1.7^{*}$ \\
\hline LIT1 & $7.2 \pm 9.4$ & $5.4 \pm 3.2$ \\
\hline MEST & $6.3 \pm 2.4$ & $6.2 \pm 1.6$ \\
\hline NESPAS & $3.4 \pm 2.2$ & $3.3 \pm 1.5$ \\
\hline PEG3 & $3.6 \pm 2.5$ & $3.0 \pm 1.8$ \\
\hline SNRPN & $3.8 \pm 2.1^{*}$ & $4.9 \pm 3.0^{*}$ \\
\hline ALU & $23.1 \pm 1.4^{* *}$ & $24.2 \pm 1.2^{* *}$ \\
\hline LINE1 & $71.2 \pm 6.1$ & $71.8 \pm 5.3$ \\
\hline
\end{tabular}

* Significant $(\mathrm{p}<0.05)$ or ${ }^{* *}$ highly significant $(\mathrm{p}<0.001)$ between-group difference.

tor infertility was assumed in males with sperm concentration fewer than $20 \mathrm{million} / \mathrm{ml}$ (oligozoospermia), fewer than $50 \%$ spermatozoa with forward progression (categories a and b) or fewer than $25 \%$ spermatozoa with category a movement (asthenozoospermia), and/or fewer than $15 \%$ spermatozoa with normal morphology (teratozoospermia).

Sperms were purified with Pure Sperm 40/80 (Nidacon, Molndal, Sweden) to remove lymphocytes, epithelial cells, cell debris, bacteria, abnormal spermatogenic cells, and seminal fluid. Sperm purity was first checked by inverted light microscopy and then confirmed by methylation analysis of BOLL, a member of the DAZ gene family, which plays a role in development of haploid germ cells [Kee et al., 2009]. The BOLL promoter is completely demethylated in sperm and fully methylated in somatic cells. Unpurified sperm samples displayed BOLL methylation values of $>30 \%$, whereas purified samples without contaminating somatic cells (by visual inspection) had around 5\%. There was no significant difference in BOLL methylation between infertile males (5.5 $\pm 2.9 \%)$ and presumably fertile controls (5.4 $\pm 2.1 \%)$ (table 1), indicating that purified sperm samples from oligospermic males are not more contaminated than those from normospermic males.

Pure sperm samples were treated with $100 \mathrm{~mm}$ TrisCl, $10 \mathrm{~mm}$ EDTA, $500 \mathrm{~mm} \mathrm{NaCl}, 1 \%$ SDS, and $2 \% \beta$-mercaptoethanol and then incubated for $2 \mathrm{~h}$ at $56^{\circ} \mathrm{C}$ with proteinase K. The DNeasy Blood and Tissue Kit (Qiagen, Hilden, Germany) was then used for DNA isolation.

\section{Infertility Treatment and ART Outcome}

A conventional high-dose exogenous follicle-stimulating hormone regimen and gonadotropin-releasing-hormone agonist co-treatment was used to induce multiple follicle growth. Oocytes were retrieved by transvaginal ultrasound-guided puncture of follicles. In vitro fertilization (IVF) and intracytoplasmatic sperm injection (ICSI) were performed according to standard protocols. The fertilization rate (of a given IVF/ICSI attempt) was calculated by the number of fertilized oocytes divided by the 
number of retrieved oocytes. Pronuclear stages were selected for further culturing on the basis of morphological criteria, i.e. pronuclear disposition and nucleolar organization [Scott et al., 2000; Nagy et al., 2003]. Prior to transfer, each embryo was graded using a systematic scoring system [Steer et al., 1992; Gardner et al., 2000]. We defined a numerical variable which indicates whether 2 or 3 top-scoring (A quality) blastocyst embryos (3 points), 1 top-scoring embryo ( 2 points), at least $1 \mathrm{~B}$-quality embryo (1 point), or only C- and/or D-quality embryos (0 points) were transferred. Clinical pregnancy was defined by the presence of a fetal sac with positive heartbeat at 6 weeks after follicular puncture. Couples who had achieved a clinical pregnancy were followed up. Twelve of the 59 clinical pregnancies had an unknown outcome, 10 ended in a spontaneous abortion and 37 led to live birth of at least 1 child.

\section{Bisulfite Pyrosequencing}

Bisulfite treatment of genomic DNA was carried out with the EpiTect Bisulfite Kit (Qiagen). Bisulfite pyrosequencing was performed on a PSQ96MA Pyrosequencing System (Biotage, Uppsala, Sweden) with the PyroGold SQA reagent kit (Biotage) [Tost et al., 2003]. Polymerase chain reaction (PCR) and sequencing primers for bisulfite pyrosequencing (table 2) were designed using the Pyrosequencing Assay Design Software (Biotage). The Pyro QCpG software (Biotage) was used for data analysis.

Gene-specific methylation assays for GTL2, H19, LIT1, MEST, NESPAS, PEG3, and SNRPN have been optimized in previous studies [Pliushch et al., 2010; Schneider et al., 2010; Zechner et al., 2010]. The studied loci represent only a small fraction of developmentally important genes. However, methylation abnormalities in these imprinted genes may be considered as indicators for more profound epigenetic defects at other loci. Therefore, we have also used assays which determine the methylation status of repetitive elements [Yang et al., 2004]. The human genome has acquired more than one million ALU repeats during the past 65 million years, including approximately 5,000 copies, which have been integrated in the past 4-6 million years after the human-chimpanzee split [Batzer and Deininger, 2002]. Our ALU assay can amplify repeats from different subfamilies, although the efficiency for evolutionarily old ALUs, which show considerable sequence variation, may be low. We estimate that at least 10,000 loci are analyzed, mainly evolutionarily young ALUs, which show less sequence variation. In addition, we used primers against the human LINE1 (L1Hs) consensus sequence to study methylation of a second interspersed repeat family. The human genome contains about 100,000 long interspersed elements that have been integrated and amplified during the last 100 million years, the majority of which are $5^{\prime}$ truncated copies of a few active L1 elements [Sheen et al., 2000]. Forward, reverse and sequencing primers are conserved in the $5^{\prime}$ region of L1Hs. In this light, our assay should amplify the majority of approximately 4,000 full-length $(6 \mathrm{~kb})$ $\mathrm{L} 1 \mathrm{Hs}$ repeats in the human genome. Our pyrosequencing assays determine the methylation status of 2 to 4 neighboring CpG sites in the amplified ALU and LINE1 repeats. Because a significant number of target $\mathrm{CpG}$ sites in the generated amplicons may be mutated, the measured average methylation value of several thousand repeats (i.e. 23-24\% ALU methylation in sperm DNA) is always lower than the true methylation. Nevertheless, it can be used as a surrogate marker for genome-wide methylation changes [Yang et al., 2004].
The mean methylation difference between duplicate measurements of the same bisulfite-converted DNA sample was $1.3 \%$ for H19 (4 samples tested), 1.7\% for GTL2 (5 samples), 1.7\% for LIT1 (9 samples), 1.0\% for MEST (3 samples), $0.4 \%$ for NESPAS (3 samples), $0.5 \%$ for PEG3 (4 samples), $0.3 \%$ for SNRPN (2 samples), $0.1 \%$ for ALU (6 samples), and $1.0 \%$ for LINE1 (3 samples). However, the main error in any methylation assay may come from differences in DNA conversion. Because the focus of this paper is on ALU methylation, we performed 2 different bisulfite treatments of 20 sperm samples, representing the whole range of ALU methylation variation $(21-26 \%)$. The technical variability was only 0.6\%: 9 duplicate measurements gave exactly the same value, 10 replicates differed by 1 percentage point and only 1 replicate differed by 2 percentage points. These results demonstrate the accuracy of our bisulfite pyrosequencing assay.

To make sure that possible systematic technical variation in sample processing does not introduce a bias to the data, the control samples and various subgroups of infertile males were analyzed in a random manner. If the endpoints of the study (i.e. pregnancy vs. no pregnancy or abortion vs. live birth) are not confounded with the experimental procedures (e.g., not all control samples are processed at 1 day), the technical variation (both systematic and random) should merely add to the overall variation of the samples.

\section{Statistical Analyses}

Quantitative methylation data were analyzed with SPSS version 17.0.1 (http://www.spss.com). Box plots were generated using the default parameters of SPSS. They display the location, dispersion and skewness of the data. The bottom and the top of the box indicate the 25 th and 75 th percentile, respectively. The $\mathrm{T}$ bars extend from the boxes to at most $1.5 \times$ the height of the box. Outliers are samples that do not lie within these T bars, extreme outliers have values more extreme than $3 \times$ the box length away from the median.

A 2-sided Mann-Whitney test was applied to detect differences in the distribution of a continuous variable between 2 groups (e.g. ALU methylation in the pregnant vs. the nonpregnant group). In addition, we plotted a receiver operating characteristics (ROC) curve, comparing the ALU methylation percentages of the 2 groups. Using the area under the curve as a test statistic for discriminative power, the corresponding $\mathrm{p}$ values were exactly those obtained by a Mann-Whitney test. A p value of less than $5 \%$ was considered significant. We derived an estimate of the odds for a favorable ART outcome (i.e. being fertile vs. infertile or pregnant vs. nonpregnant), given a certain ALU methylation value. This was achieved by fitting a smooth kernel density estimator to the empirical ALU methylation distributions of the respective groups. Then, for the regions in which sufficient samples from both groups existed (21-25\% ALU methylation), the log2 quotient of the 2 densities was calculated. In order to obtain confidence bands, we repeated this procedure with 1,000 bootstrap samples of the data and calculated the central 95\% interval of the obtained values.

The Spearman's rank correlation test was used to test for correlations between sperm DNA methylation and sperm quality parameters. The correlation coefficient $r$ can range from -1 (negative correlation) to +1 (positive correlation). The corresponding $\mathrm{p}$ value determines whether or not $r$ significantly differs from 0 (no correlation). 
Table 2. Genes and primers for bisulfite pyrosequencing

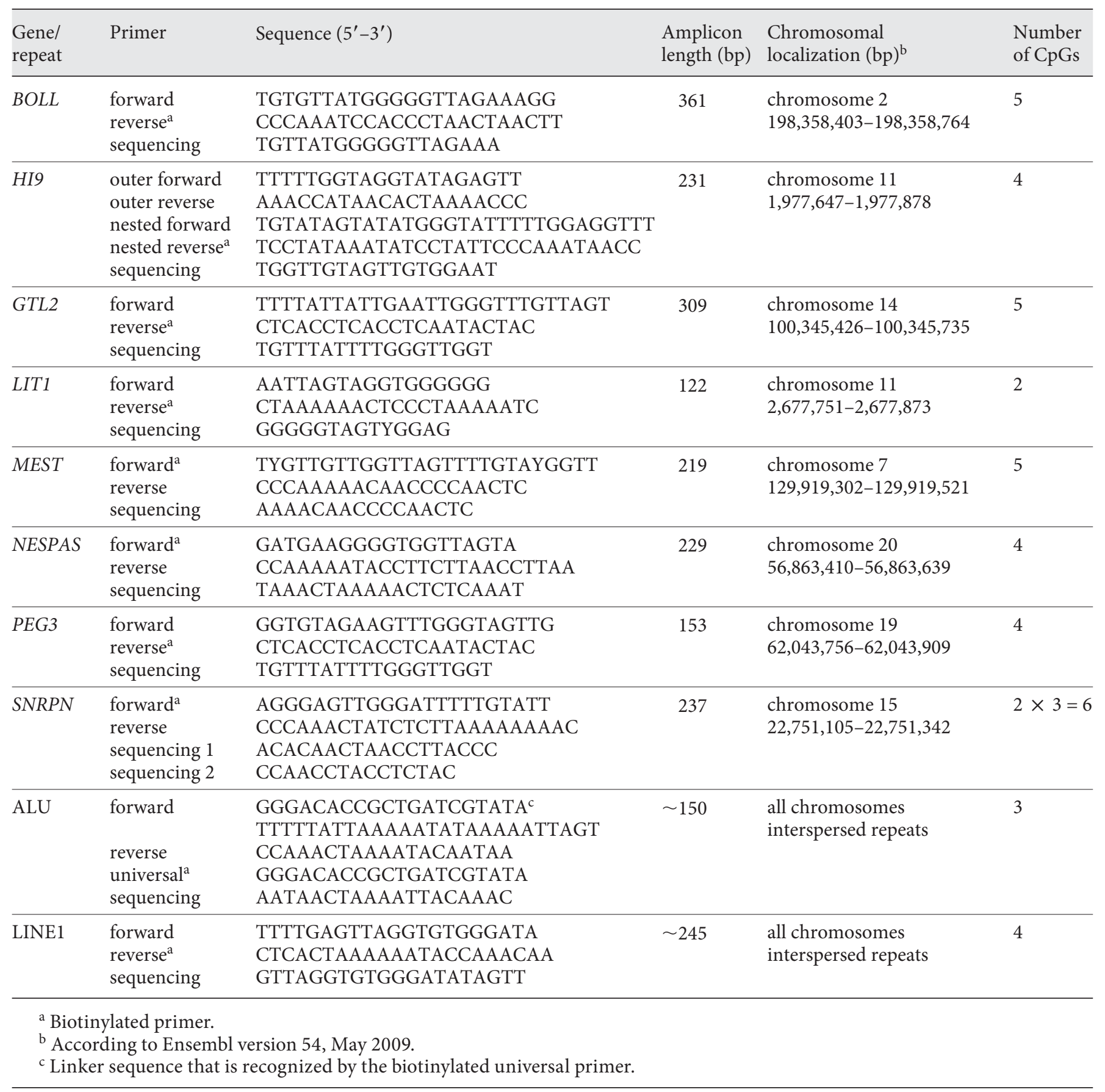

A Fisher linear discriminant analysis including the methylation data sets of 7 imprinted genes and 2 repeats, as well as a univariate discrimination analysis based on ALU methylation alone, were performed to assess the predictive power of ALU. We additionally calculated logistic regression analyses of the 4 endpoints (male fertility, pregnancy, abortion, and live birth) against all clinical covariates and ALU and LINE1 methylation. We applied backward elimination to sequentially reduce the number of variables while retaining the prediction accuracy for ART outcome. This procedure generates a minimal model that is supposed to contain only variables that cannot satisfactorily be explained by other variables in the model. 


\section{Results}

\section{Association of Sperm DNA Methylation with Male} Infertility

The germline DMRs of 2 paternally (H19 and GTL2) and 5 maternally methylated (LIT1, MEST, NESPAS, $P E G 3$, and SNRPN) imprinted genes, as well as of ALU and LINE1 repeats, were analyzed by bisulfite pyrosequencing in 141 sperm samples, 106 from males with abnormal and 35 from males with normal variables in semen analyses. Male factors but no detectable female factors impairing fertility were present in 59 couples attempting pregnancy. Combined male and female factors accounted for 47 couples. In essentially all cases of strictly male or combined infertility, oocytes were injected with single sperm by ICSI. Neither male nor female factors were found in 7 couples. In 28 couples, the males displayed normal semen parameters (in repeated analyses) and no other cause of male infertility, but there was clear indication for female factors, in particular tubal infertility. These 28 sperm samples from presumably fertile males attending a fertility center were taken as controls. In most cases of strictly female-factor infertility, oocytes were fertilized by conventional IVF.

Two imprinted genes, GTL2 and SNRPN showed minor, but significant (2-sided Mann-Whitney test; $\mathrm{p}<$ 0.05) methylation differences between the 106 sperm samples from infertile males and the 28 presumably fertile controls (table 1). ALU methylation was significantly (Mann-Whitney test; $\mathrm{p}<0.001)$ lower in infertile males $(23.1 \pm 1.4 \%)$ than in controls $(24.2 \pm 1.2 \%)$ (fig. 1A). When we calculated a Spearman rank correlation of all analyzed samples and loci, SNRPN and ALU methylation were significantly ( $\mathrm{p} \leq 0.01$ and $\mathrm{p}<0.001$, respectively) correlated with sperm count (correlation coefficient was 0.35 for SNRPN and 0.60 for ALU), percentage of motile sperm ( 0.31 and 0.46 , respectively) and percentage of sperm with normal morphology (0.21 and 0.49).

\section{Aberrant Methylation of Imprinted Genes}

The box plots in figure 2 show the distribution of methylation values for each studied gene and repeat in the 141 analyzed sperm samples. Methylation values falling in the $\mathrm{T}$ bars were considered normal. Outliers and extreme outliers in imprinted genes may indicate aberrant methylation imprints that imply errors in establishment and/or maintenance of the appropriate germline methylation patterns in a subset of sperms. Thirty-one of the $141(22 \%)$ sperm samples displayed at least 1 outlier in an imprinted gene, including 19 (14\%) samples with at least 1 extreme outlier and 4 (3\%) samples with methylation abnormalities in multiple genes. Most outliers were found in LIT1 (11 samples) and PEG3 (10 samples). Aberrant methylation imprints occurred significantly ( $\chi^{2}$ test; $\mathrm{p}=0.03)$ more frequently in sperm samples from infertile males $(28 / 106 ; 26 \%)$ than in controls $(2 / 28 ; 7 \%)$. Sperm samples with aberrant methylation imprints had a significantly lower sperm count (2-sided Mann-Whitney test; $\mathrm{p}=0.01$ for samples with extreme outliers and $\mathrm{p}=$ 0.06 for samples with outliers), lower sperm motility ( $\mathrm{p}=$ 0.02 and $p=0.06$, respectively) and lower proportion of sperm with normal morphology $(\mathrm{p}=0.001$ and $\mathrm{p}=0.01)$ than samples with normal methylation imprints. However, the fertilization rate and ART outcome did not differ significantly between sperm samples with normal versus abnormal methylation imprints.

\section{ALU Methylation and ART Outcome}

Compared to LINE1 (71.3 $\pm 5.9 \%)$, ALU repeats showed a rather narrow range of methylation variation $(23.4 \pm 1.4 \%)$ in the 141 analyzed sperm samples (table 1). In our box-blot analysis (fig. 2), 8 samples presented with outliers in ALU methylation. Although the numbers are low, it is interesting to note that 3 of 4 hypermethylated outliers, but none of the 4 hypomethylated outliers, established a pregnancy.

Following ICSI, 44 of the 106 sperm samples from infertile males led to pregnancy. Maternal age (33.3 vs. 35.6 years) and embryo quality ( 2.4 vs. 2.1 in our scoring system) were significantly different (2-sided Mann-Whitney test; $\mathrm{p}<0.05)$ between the pregnant and the nonpregnant group. Paternal age (37.1 vs. 39.1 years) showed a trend $(\mathrm{p}=0.056)$ difference. The fertilization rate (82.4 vs. $80.7 \%$ ) was comparable in both groups. Of the studied imprinted genes, only GTL2 showed a minor but significant $(\mathrm{p}=0.03)$ between-group methylation difference. There was a significant (ROC curve, Mann-Whitney test; $\mathrm{p}<0.01)$ difference in ALU methylation between sperm samples leading to pregnancy $(23.6 \pm 1.2 \%)$ and those that did not (22.8 $\pm 1.5 \%)$ (fig. $1 \mathrm{~B})$.

Twenty-seven of the 44 pregnancies from infertile males led to live birth of a baby, 7 ended in a spontaneous abortion and 10 had an unknown outcome. Again, ALU methylation was significantly (ROC curve; $\mathrm{p}<0.001$ ) higher in sperm samples leading to birth $(24.1 \pm 0.9 \%)$ than in those that did not $(22.7 \pm 1.1 \%)$ (fig. 1C). In contrast, sperm samples leading to spontaneous abortion had a significantly (ROC curve; $\mathrm{p}=0.027$ ) lower ALU methylation $(22.1 \pm 1.2 \%)$ than those leading to live birth (24.1 \pm 0.9\%) (fig. 1D). Parental age, fertilization rate, 
Fig. 1. ALU hypomethylation has a negative impact on ART outcome. A Bar diagrams showing the distribution of ALU methylation values in infertile males (black bars) and presumably fertile controls (gray bars). In the middle, the corresponding ROC curve is presented. The area under the curve indicates the degree of methylation difference between groups. On average, the ALU methylation values were $1.1 \%$ higher in the control group. The panel on the right side shows the log2 odds for a favorable outcome (i.e. being fertile vs. infertile), plotted as a function of the methylation percentage. Shown are the $95 \%$ bootstrap confidence intervals. B ALU methylation values in sperm samples from infertile males leading to pregnancy (gray bars) and those not leading to pregnancy (black bars). On average, the ALU methylation values were $0.8 \%$ higher in the pregnancy group. C ALU methylation values in sperm samples from infertile males leading to live birth of a baby (gray bars) and those not leading to birth (black bars). On average, the ALU methylation values were $1.4 \%$ higher in the live-birth group. D ALU methylation values in sperm samples from infertile males leading to live birth (gray bars) and those leading to spontaneous abortion (black bars). On average, the ALU methylation values were $2.0 \%$ higher in the baby-take home group than in the abortion group.
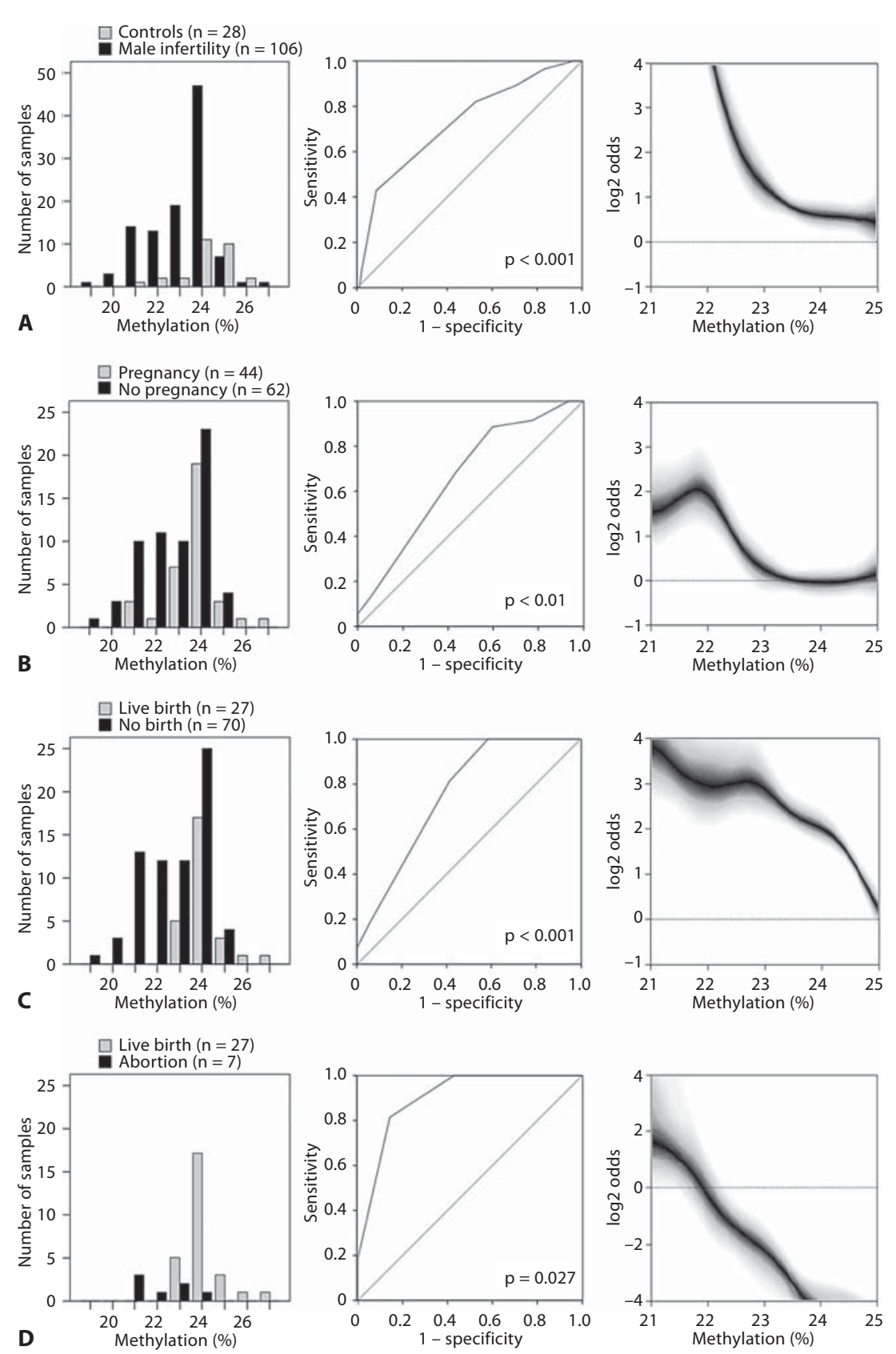

embryo quality, and imprinted gene methylation did not differ significantly between the baby-take home group, the group without a baby and the abortion group; hence, there was no need to correct for these factors. In the 7 spontaneously aborted pregnancies, the classical parameters even appeared to be somewhat more favorable than in the live-birth group (maternal age 32.9 vs. 33.3 years, paternal age 34.4 vs. 38.2 years, fertilization rate 79 vs. $82 \%$, and embryo quality 2.9 vs. 2.3 ), suggesting that other risk factors (i.e. disturbances in the sperm epigenome) may be more important.

The dependence of ART outcome on methylation percentage was further illustrated by the $\log 2$ odds for a favorable outcome (fig. 1, right panels). In all 4 between- 


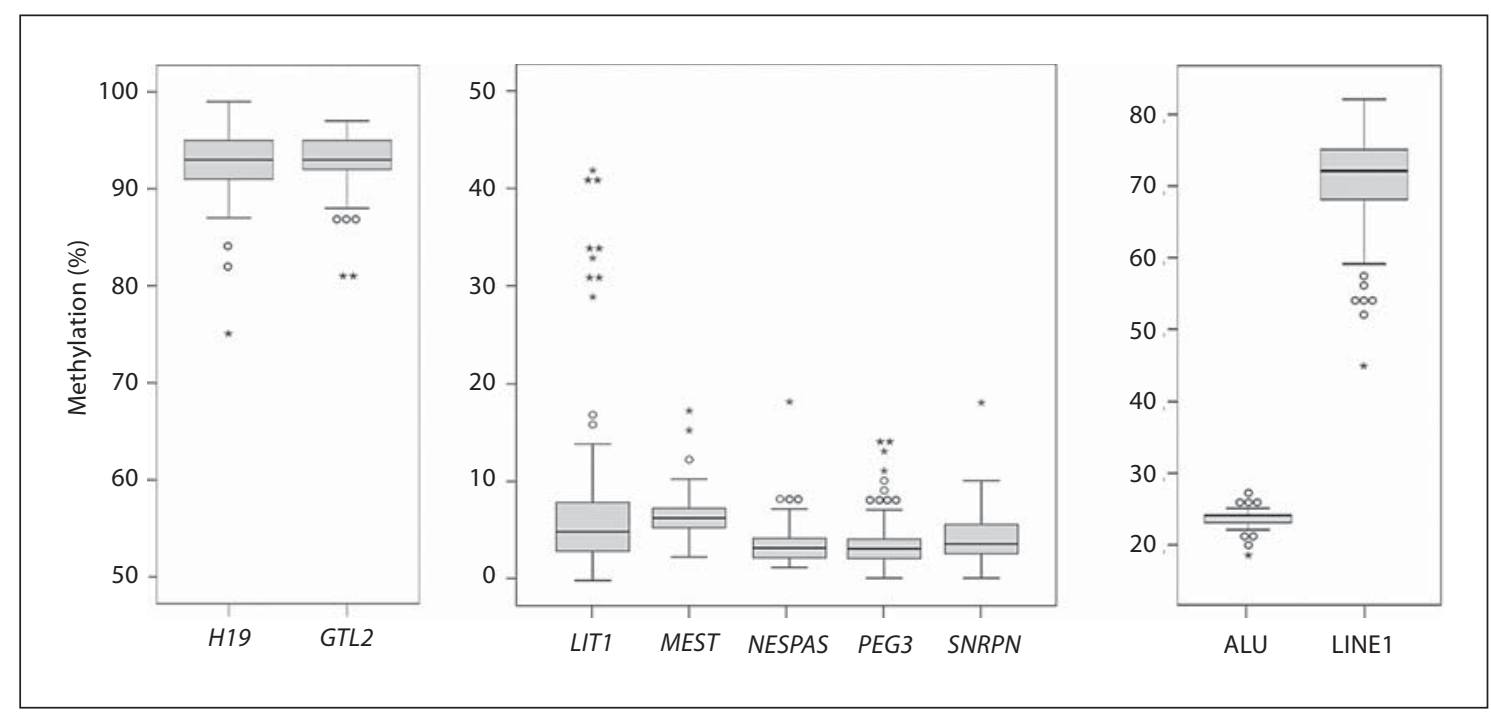

Fig. 2. Box plots showing the distribution of methylation values of 2 paternally methylated (H19 and GTL2) and 5 maternally methylated (LIT1, MEST, NESPAS, PEG3, and SNRPN) imprinted genes as well as of 2 (ALU and LINE1) repetitive elements in 141 IVF/ICSI sperm samples. The median is represented by a horizontal line. The bottom of the box indicates the 25 th percentile, the top the 75 th percentile. Outliers are shown as circles and extreme outliers as stars.

group comparisons (presumable fertile vs. infertile, pregnant vs. nonpregnant, live birth vs. no live birth, and live birth vs. abortion), a decrease in ALU methylation was clearly coupled to an increase in the odds for an unfavorable prognosis/ART outcome.

\section{Assessing the Dependence of ART Outcome on Sperm DNA Methylation}

A multivariate discrimination analysis including the methylation values of all analyzed genes and repeats was calculated to find out whether or not sperm DNA methylation can distinguish between infertile and presumably fertile males. GTL2, PEG3 and ALU methylation were the remaining variables with the highest predictive power. In $80 \%$ of the analyzed cases, they predicted whether the sperm sample was derived from an infertile male or the control group. The odds ratio (OR) of the corresponding contingency table was 5.7 (95\% CI 1.2, 26.3). In a discrimination analysis based on ALU methylation alone, $79 \%$ of the samples were classified correctly (OR $=4.0 ; 95 \%$ CI $0.5,29.8)$. To assess the influence of sperm DNA methylation on the ability of ART to establish pregnancy in couples with male-factor or combined infertility, we performed a multivariate discrimination analysis based on the methylation values of the 106 sperm samples from infertile males. ALU and LINE1 methylation were identi- fied as the variables with the highest predictive power, $71 \%(\mathrm{OR}=5.0 ; 95 \%$ CI 1.9, 13.1). When only ALU was used for prediction, the power was $60 \%(\mathrm{OR}=2.5$; $95 \%$ CI 1.1, 5.6). A multivariate discrimination analysis for prediction of live birth of a baby following ART with a sperm sample from an infertile male, including the methylation values of all analyzed genes and repeats, revealed ALU methylation as the only variable of significant influence. It correctly predicted ART outcome in $73 \%$ of cases $(\mathrm{OR}=3.8 ; 95 \% \mathrm{CI} 0.9,15.2)$. When only considering ICSI pregnancies with sperm samples from infertile males, ALU methylation correctly predicted for $89 \%$ of the couples whether the pregnancy ended in a spontaneous abortion or led to birth (OR $=27$; 95\% CI 2.2, 324.9).

The results of the logistic regression of ART endpoints against all clinical covariates and ALU and LINE1 methylation confirmed that the effect of ALU methylation could not be explained through other covariates (male or female age, number of retrieved oocytes, number of fertilized oocytes, embryo quality, sperm shape, sperm count, sperm motility, and LINE1 methylation). For the 3 endpoints pregnancy, abortion and live birth among all variables, ALU methylation had the most significant influence in the initial model, and it turned out to be the most significant factor after applying backward elimination $(\mathrm{p}<0.003$; generally, the variables female age and 
LINE1 methylation were the only covariates that remained in the minimal model). Collectively, these data suggest that ALU hypomethylation is not only associated with male infertility and/or poor sperm quality, but more importantly also with a poor ART outcome.

\section{Discussion}

A considerable proportion ( $>20 \%$ ) of IVF/ICSI sperm samples in our study exhibited aberrant methylation imprints. These samples were characterized by low sperm count, reduced sperm motility and abnormal sperm morphology. However, the most important question of couples undergoing infertility treatment is whether or not they can achieve a pregnancy and live birth of a baby. Although a number of conceptually related studies [Marques et al., 2004, 2008; Houshdaran et al., 2007; Kobayashi et al., 2007, 2009; Boissonnas et al., 2010; Hammoud et al., 2010; Poplinski et al., 2010] have shown similar associations between abnormal methylation imprints and low sperm quality, the effects on ART outcome remained unclear. Kobayashi et al. [2007] found imprinting defects in 24 of 97 oligospermic males and only 2 of these 24 patients achieved a pregnancy by ART. Unfortunately, there were no data on the pregnancy rate in the control group with normal imprinting patterns. Consistent with our study, Boissonnas et al. [2010] did not find a significant effect of abnormal methylation imprints in sperm samples of infertile men on pregnancy rate. Benchaib et al. [2005] used immunofluorescent staining with an anti-5methylcytosine antibody to classify sperm samples according to their global methylation level. When sperm DNA methylation was $>555$ arbitrary units, the pregnancy rate was significantly higher than in the lower $(<555)$ group. However, methylcytosine staining intensity can be influenced by many factors, and, therefore, immunofluorescence assays are difficult to standardize.

The average methylation levels of several thousand ALU or LINE1 repeats, as determined by bisulfite pyrosequencing, can serve as sensitive markers for genomewide epigenetic changes [Yang et al., 2004], even though they are certainly not a perfect representation of the entire genome. In sperm, ALU repeats showed a much smaller (natural and/or technical) range of methylation variation than LINE1 repeats. This might explain the high predictive power of ALU methylation values in our study, compared to LINE1 methylation. On the other hand, the mechanism(s) controlling the methylation of ALU repeats in the male germline may differ from LINE1 and other repetitive or nonrepetitive sequences classes. Repetitive elements appear to influence spreading and/or maintenance of $\mathrm{X}$ inactivation. LINE1 repeats are significantly enriched around transcription start sites of $\mathrm{X}$-chromosomal genes that are subject to inactivation, whereas ALU repeats are enriched in genes that escape inactivation [Carrel et al., 2006; Wang et al., 2006]. In the early mouse embryo [Thorvaldsen et al., 2006] and most likely also in human preimplantation embryos [van den Berg et al., 2009], the paternal X chromosome is selectively inactivated. This imprinted $\mathrm{X}$ inactivation precedes random $\mathrm{X}$ inactivation in the soma, and, at least in the mouse, it continues through the placental lineages. Disturbances in dosage compensation in female embryos are not compatible with normal development. It is tempting to speculate that abnormal repeat methylation and/or chromatin structure of sperm chromosomes interferes with $\mathrm{X}$ inactivation and/or other epigenetic key regulatory mechanisms, which are essential for the establishment and/or maintenance of pregnancy. In this context, it is interesting to note that the majority of genes contain 1 or several ALUs in close proximity $5^{\prime}$ to CpG islands in cis-regulatory regions in promoters and/or first exons [International Human Genome Sequencing Consortium, 2004]. For several genes, it has been demonstrated that ALU sequences are involved in the regulation of transcription [Hamdi et al., 2000; Ludwig et al., 2005].

Several lines of evidence suggest that ALU methylation (rather than methylation abnormalities in individual genes) may have an impact on ART outcome in malefactor infertility. (i) ALU methylation in sperm samples of infertile males was approximately $1 \%$ lower (23.1 vs. $24.2 \%$ ) than in presumably fertile males. (ii) Sperm samples from infertile males leading to pregnancy and live birth displayed higher ALU methylation levels (23.6 and $24.1 \%$, respectively) than those failing to establish pregnancy $(22.8 \%)$ or to carry a child to term $(22.7 \%)$. It is reassuring that on average infertile males with a favorable ART outcome showed ALU methylation values closely resembling fertile controls, whereas infertile males with poor pregnancy prognosis displayed ALU hypomethylation. (iii) There was a remarkable $2 \%$ difference in the methylation levels of sperm samples from infertile males leading to spontaneous abortion (22.1\%) versus samples leading to birth (24.1\%). This supports the idea that epigenetic abnormalities may contribute to the high rate of unexplained pregnancy loss in humans. Recently, we proposed a multifactorial threshold model for pregnancy loss [Pliushch et al., 2010; Zechner et al., 2010]. If the number of epimutations affecting developmentally im- 
portant genes exceeds a critical threshold, the phenotype (spontaneous abortion) may become manifest. Similar to other multifactorial diseases, additional genetic and environmental factors may also play a role.

Our statistical analyses suggest that ALU hypomethylation is prognostic for poor ART outcome. The high reproducibility of our measurements (0.6 percentage points for ALU repeats) reduces within-group variation. Yet, our testing procedures do not assume or require a low technical variability, and significance calls remain valid irrespective of the actual technical variability. The within-group variation is scarcely smaller $(0.9-1.5$ percentage points) than the between-group difference (0.82.0 percentage points), and in any group a significant proportion of samples exhibits methylation values of 23 or $24 \%$. Therefore, it is difficult to predict ART outcome for a given sperm sample falling in this range of methylation variation. At this point our study is mainly exploratory. Before clinical application, additional studies are needed to test whether or not sperm methylation analysis can add something to classical semen analysis. More sophisticated algorithms including other epigenetic and clinical parameters may increase the predictive power. Similar to conventional semen parameter analysis, methylation analysis of different sperm samples from the same male may also improve the prognostic correlation with pregnancy outcome. Overall, bisulfite pyrosequencing is a highly accurate high-throughput technique for quantitative methylation analysis [Tost et al., 2003; Schneider et al., 2010]. Furthermore, our findings may open new strategies to explore the possibility of restoring fertility in male subfertility/infertility cases by modulating DNA methylation (e.g. by methyl donor nutrition or drugs).
Our results suggest that epigenetic signatures in sperm DNA are associated with factors that may influence the outcome of male infertility treatment. The most dramatic changes in chromatin structure and higher-order nuclear organization occur shortly after fertilization. Sperm-egg fusion activates the oocyte which then remodels the almost crystalline, mostly protamine-packaged sperm chromatin into a functional histone-packaged male pronucleus [Perrault, 1992; Haaf, 2006]. The paternal genome is actively demethylated in the zygote, whereas the maternal genome undergoes gradual passive demethylation by a replication-dependent mechanism in embryo cleavage stages [Mayer et al., 2000]. Although early mammalian development appears to be almost exclusively under maternal control, functional organization of sperm chromatin is assumed to be important for the initiation and regulation of paternal gene activity in the early embryo. Sperm chromatin may be organized in a structure that determines a specific required pattern of unpackaging and expression of the paternal genome upon fertilization [Haaf and Ward, 1995; Miller et al., 2010]. It is well known that changes in DNA methylation are associated with changes in chromatin structure [Haaf, 1995; Jaenisch and Bird, 2003]. The quantification of ALU methylation may reflect changes in sperm chromatin packaging that directly or indirectly influence paternal chromosome and gene functions in the early embryo.

\section{Acknowledgements}

We thank Prof. Hans Zischler for help with the ALU and LINE1 primers. This work was supported by research grants (FOR 1041 and SFB 646) from the German Research Foundation. A.T. was supported by an LMU guest professorship.

\section{References}

Bartolomei MS, Tilghman SM: Genomic imprinting in mammals. Annu Rev Genet 31: 493-525 (1997).

Batzer MA, Deininger PL: Alu repeats and human genomic diversity. Nat Rev Genet 3: 370-379 (2002).

Benchaib M, Braun V, Ressnikof D, Lornage J, Durand P, et al: Influence of global sperm DNA methylation on IVF results. Hum Reprod 20:768-773 (2005).

Boissonnas CC, Abdalaoui HE, Haelewyn V, Fauque P, Dupont JM, et al: Specific epigenetic alterations of IGF2-H19 locus in spermatozoa from infertile men. Eur J Hum Genet 18:73-80 (2010)
Carrel L, Park C, Tyekucheva S, Dunn J, Chiaromonte F, Makova KD: Genomic environment predicts expression patterns on the human inactive X chromosome. PLoS Genet 2:e151 (2006).

Gardner DK, Lane M, Stevens J, Schlenker T, Schoolcraft WB: Blastocyst score affects implantation and pregnancy outcome: towards a single blastocyst transfer. Fertil Steril 73: 1155-1158 (2000).

Haaf T: The effects of 5-azacytidine and 5-azadeoxycytidine on chromosome structure and function: implications for methylationassociated cellular processes. Pharmac Ther 65:19-46 (1995).
Haaf T: Methylation dynamics in the early mammalian embryo: implications of genome reprogramming defects for development. Curr Top Microbiol Immunol 310:13-22 (2006).

Haaf T, Ward DC: Higher-order nuclear structure in mammalian sperm revealed by in situ hybridization and extended chromatin fibers. Exp Cell Res 219:604-611 (1995).

Hagan CR, Sheffield RF, Rudin CM: Human Alu element retrotransposition induced by genotoxic stress. Nat Genet 35:219-220 (2003).

Hajkova P, Erhardt S, Lane N, Haaf T, El-Maarri $\mathrm{O}$, et al: Epigenetic reprogramming in mouse primordial germ cells. Mech Dev 117:15-23 (2002). 
Hamdi HK, Nishio H, Tavis J, Zielinski R, Dugaiczyk A: Alu-mediated phylogenetic novelties in gene regulation and development. J Mol Biol 299:931-939 (2000).

Hammoud SS, Purwar J, Pflueger C, Cairns BR, Carrell DT: Alterations in sperm DNA methylation patterns at imprinted loci in two classes of infertility. Fertil Steril 94:17281733 (2010).

Houshdaran S, Cortessis VK, Siegmund K, Yang A, Laird PW, Sokol RZ: Widespread epigenetic abnormalities suggest a broad DNA methylation erasure defect in abnormal human sperm. PLoS One 2:e1289 (2007).

International Human Genome Sequencing Consortium: Finishing the euchromatic sequence of the human genome. Nature 431: 931-945 (2004).

Jaenisch R, Bird A: Epigenetic regulation of gene expression: how the genome integrates intrinsic and environmental signals. Nat Genet 33 Suppl:245-254 (2003).

Kee K, Angeles VT, Flores M, Nguyen HN, Reijo Pera RA: Human DAZL, DAZ and BOULE genes modulate primordial germ-cell and haploid gamete formation. Nature 462:222225 (2009)

KellyTL,LiE, TraslerJM:5-aza-2'-deoxycytidine induces alterations in murine spermatogenesis and pregnancy outcome. J Androl 24: 822-830 (2003).

Kobayashi H, Sato A, Otsu E, Hiura H, Tomatsu $\mathrm{C}$, et al: Aberrant DNA methylation of imprinted loci in sperm from oligospermic patients. Hum Mol Genet 16:2542-2551 (2007).

Kobayashi H, Hiura H, John RM, Sato A, Otsu E, et al: DNA methylation errors at imprinted loci after assisted conception originate in the parental sperm. Eur J Hum Genet 17:15821591 (2009).

Li E: Chromatin modification and epigenetic reprogramming in mammalian development. Nat Rev Genet 3:662-673 (2002).

Ludwig A, Rozhdestvensky TS, Kuryshev VY, Schmitz J, Brosius J: An unusual primate locus that attracted two independent Alu insertions and facilitates their transcription. J Mol Biol 350:200-214 (2005).

Marchal R, Chicheportiche A, Dutrillaux B, Bernardino-Sgherri J: DNA methylation in mouse gametogenesis. Cytogenet Genome Res 105:316-324 (2004).
Marques CJ, Carvalho F, Sousa M, Barros A: Genomic imprinting in disruptive spermatogenesis. Lancet 363:1700-1702 (2004).

Marques CJ, Costa P, Vaz B, Carvalho F, Fernandes $S$, et al: Abnormal methylation of imprinted genes in human sperm is associated with oligozoospermia. Mol Hum Reprod 14: 67-74 (2008).

Mayer W, Niveleau A, Walter J, Fundele R, Haaf T: Demethylation of the zygotic paternal genome. Nature 403:501-502 (2000).

Miller D, Brinkworth M, Iles D: Paternal DNA packaging in spermatozoa: more than the sum of its parts? DNA, histones, protamines and epigenetics. Reproduction 139:287-301 (2010).

Nagy ZP, Dozortsev D, Diamond M, Rienzi L, Ubaldi F, et al: Pronuclear morphology evaluation with subsequent evaluation of embryo morphology significantly increases implantation rates. Fertil Steril 80:67-74 (2003).

Oakes CC, La Salle S, Smiraglia DJ, Robaire B, Trasler JM: Developmental acquisition of genome-wide DNA methylation occurs prior to meiosis in male germ cells. Dev Biol 307: 368-379 (2007).

Perrault SD: Chromatin remodeling in mammalian zygotes. Mutat Res 296:43-55 (1992).

Pliushch G, Schneider E, Weise D, El Hajj N, Tresch A, et al: Extreme methylation values of imprinted genes in human abortions and stillbirths. Am J Pathol 176:1084-1090 (2010).

Poplinski A, Tüttelmann F, Kanber D, Horsthemke B, Gromoll J: Idiopathic male infertility is strongly associated with aberrant methylation of MEST and IGF2/H19 ICR1. Int J Androl 33:642-649 (2010).

Raman R, Narayan G: 5-Azadeoxycytidine-induced inhibition of differentiation of spermatogonia into spermatocytes in the mouse. Mol Reprod Dev 42:284-290 (1995).

Reik W, Dean W, Walter J: Epigenetic reprogramming in mammalian development. Science 293:1089-1093 (2001).

Reik W, Constância M, Fowden A, Anderson N, Dean W, et al: Regulation of supply and demand for maternal nutrients in mammals by imprinted genes. J Physiol 547:35-44 (2003).

Rubin CM, van de Voort CA, Teplitz RL, Schmid CW: Alu repeated DNAs are differentially methylated in primate germ cells. Nucleic Acids Res 22:5121-5127 (1994).

Schmid CW: Does SINE evolution preclude Alu function? Nucleic Acids Res 26:4541-4550 (1998).
Schneider E, Pliushch G, El Hajj N, Galetzka D, Puhl A, et al: Spatial, temporal and interindividual epigenetic variation of functionally important DNA methylation patterns. Nucleic Acids Res 38:3880-3890 (2010).

Scott L, Alvero R, Leondires M, Miller B: The morphology of human pronuclear embryos is positively related to blastocyst development and implantation. Hum Reprod 15: 2394-2403 (2000).

Sheen FM, Sherry ST, Risch GM, Robichaux M, Nasidze I, et al: Reading between the LINEs: human genomic variation induced by LINE1 retrotransposition. Genome Res 10:14961508 (2000).

Steer CV, Mills CL, Tan SL, Campell S, Edwards RG: The cumulative embryo score: a predictive embryo scoring technique to select the optimal number of embryos to transfer in an in-vitro fertilization and embryo transfer programme. Hum Reprod 7:117-119 (1992).

Thorvaldsen JL, Verona RI, Bartolomei MS: Xtra! X-tra! News from the mouse X chromosome. Dev Biol 298:344-353 (2006).

Tost J, Dunker J, Gut IG: Analysis and quantification of multiple methylation variable positions in $\mathrm{CpG}$ islands by pyrosequencing. Biotechniques 35:152-156 (2003).

Van den Berg IM, Laven JS, Stevens M, Jonkers I, Galjaard RJ, et al: X chromosome inactivation is initiated in human preimplantation embryos. Am J Hum Genet 84:771-779 (2009).

Wang Z, Willard HF, Mukherjee S, Furey TS: Evidence of influence of genomic DNA sequence on human $\mathrm{X}$ chromosome inactivation. PLoS Comput Biol 2:e113 (2006).

Whitelaw E, Martin DI: Retrotransposons as epigenetic mediators of phenotypic variation in mammals. Nat Genet 27:361-365 (2001).

Yang AS, Estécio MR, Doshi K, Kondo Y, Tajara $\mathrm{EH}$, Issa JP: A simple method for estimating global DNA methylation using bisulfite PCR of repetitive DNA elements. Nucleic Acids Res 32:e38 (2004).

Yoder JA, Walsh CP, Bestor TH: Cytosine methylation and the ecology of intragenomic parasites. Trends Genet 13:335-340 (1997).

Zechner U, Pliushch G, Schneider E, El Hajj N, Tresch A, et al: Quantitative methylation analysis of developmentally important genes in human pregnancy losses after ART and spontaneous conception. Mol Hum Reprod 16:704-713 (2010). 